\title{
A Modular 2-DOF Force-Sensing Instrument for Laparoscopic Surgery
}

\author{
Srinivas K. Prasad ${ }^{1,3}$, Masaya Kitagawa ${ }^{1}$, Gregory S. Fischer ${ }^{1}$, Jason Zand ${ }^{2}$, \\ Mark A. Talamini' ${ }^{2}$, Russell H. Taylor ${ }^{1}$, and Allison M. Okamura ${ }^{1}$ \\ ${ }^{1}$ Johns Hopkins University \\ Engineering Research Center for Computer Integrated Surgical Systems and Technology \\ 315 New Engineering Building, 3400 N. Charles Street, Baltimore, MD 21218 \\ \{srini, mkitagawa, gfisch, rht, aokamura\} @cs.jhu.edu, \\ http://cisstweb.cs.jhu.edu \\ ${ }^{2}$ Johns Hopkins Medical Institutions, Department of Surgery \\ Blalock 665, 600 N. Wolfe Street, Baltimore, MD 21287 \\ \{jzand, talamini\}@jhmi.edu \\ ${ }^{3}$ Washington University School of Medicine, Department of Neurological Surgery \\ 660 South Euclid Avenue, Campus Box 8057, St. Louis, MO 63110
}

\begin{abstract}
Minimally Invasive Surgery (MIS) has enjoyed increasing attention and development over the last two decades. As MIS systems evolve, the surgeon is increasingly insulated from patient contact, creating a trade-off between surgical sensory information and patient invasiveness. Incorporation of haptic feedback into MIS systems promises to restore sensory information surrendered in favor of minimal invasiveness. We have developed a novel, biocompatible 2DOF force-sensing sleeve that can be used modularly with a variety of $5 \mathrm{~mm}$ laparoscopic instruments. The functional requirements for such a device are defined, and design strategies are explored. Our formal device design is outlined and device calibration is presented with derived calibration functions. Illustrative experimental force data from a porcine model is presented. This device can be used for intra-abdominal force recording and feedback in laparoscopic environments; the implications and future potential for this technology are explored.
\end{abstract}

\section{Introduction}

\subsection{Background}

Minimally Invasive Surgery (MIS) has enjoyed increasing attention and development over the last two decades. Decreased patient morbidity, increased recovery rates and shorter hospital stays are all benefits that have fueled the popularity of MIS procedures. However, MIS procedures are often more technically demanding and constrained than open procedures. There is a significant loss of tactile and sometimes visual information that precludes use in more information-intensive surgical procedures. As minimally invasive surgical systems evolve, the surgeon is increasingly insulated from patient contact, creating a trade-off between surgical sensory infor- 
mation and patient invasiveness. The loss of haptic (force and tactile) information is costly in terms of both increased operative time and decreased surgeon dexterity. Moreover, as robotic systems are introduced into laparoscopic environments, surgeons are enjoying significantly improved dexterity while preserving the minimal invasiveness of laparoscopic surgery. This evolution of MIS has further decreased the haptic information available to operators; while haptic information is simply attenuated in conventional laparoscopic surgery, it is altogether absent in the telesurgical systems available today. For these systems to achieve their fullest potential, it is imperative that they create telepresence.

In addition to providing a more realistic physical interaction, haptic feedback systems offer several other clinically valuable benefits. For teleoperation systems, we could implement force scaling to enhance force resolution in microsurgery and measure surgical skill or procedural complexity. In addition, we could impose force limits in robotic environments by collecting typical force levels applied during surgery. For freehand systems, sensory substitution for force feedback through auditory or visual signals is possible. Incorporation of haptic feedback into evolving MIS systems promises to improve patient safety and restore sensory information surrendered in favor of minimal invasiveness.

The larger goal of this project is to quantify intra-abdominal forces administered by laparoscopic instruments during MIS. Accordingly, the primary objective is to design and develop a biocompatible instrument capable of measuring applied forces within the normal operative force range. We focus on the application of haptic sensing to freehand devices. A secondary objective of this project is to develop a graphical user interface necessary to record and display force information from the device. In an effort to characterize sensory substitution modalities, this GUI is equipped with provisions for acoustic feedback. To make meaningful contributions to MIS, it is imperative that force information be collected during surgery. We have developed a biocompatible device with the belief that, by making force collection safe and accurate for use in humans, we can significantly improve MIS procedures.

\subsection{Prior Work}

Several research labs have developed platforms for collecting intra-operative force information. While a few of these devices have targeted "open" surgical environments [4,7], most have explored the value of haptics in the MIS environment [1-3,10-15]. Most of the prior devices were instrumented with elaborate force sensors at the instrument handles to ease design and regulatory constraints [3,5,8,10-13]. It is unlikely that force information derived from open procedures extends to more constrained laparoscopic procedures, reinforcing the value of intra-abdominal force sensing during laparoscopy.

A significant amount of research has been dedicated to haptic information display mechanisms. Usually, this is explored by developing full systems for haptic collection and display $[9,15]$. Moreover, these systems have been used to characterize the kinematics and dynamics of surgery and the evaluation of surgical skills [6,8-10,12-14].

In past years, many robotic and teleoperation systems have been developed to surmount the challenges of laparoscopic surgery. By decoupling information collection and display, interfaces can be independently optimized for both the surgeon and 
the patient. These robotic systems are ideal platforms for evaluating the importance of haptic feedback since they offer a mechanism for intuitive force display.

\section{Device Design}

\subsection{Functional Requirements}

Before designing our device, we delineated a number of functional requirements that we believe to be essential for collecting clinically meaningful intra-abdominal human surgical force data. Each requirement is outlined below with an overview of our solution.

1. Modularity: We fabricated a force-sensing sleeve to fit concentrically over an existing device shaft. This sleeve ensures compliance with any $5 \mathrm{~mm}$ stainless steel laparoscopic device. Tight tolerances between the shaft and sleeve aim to allow the approximation of a single solid stainless steel beam for bending calculations.

2. Biocompatibility: The device shaft is constructed with Surgical Stainless Steel grade 304, which is covered with a Teflon ${ }^{\circledR}$ sleeve. All joints and interfaces are sealed with Biocompatible USP Class-VI epoxy. A biocompatible, fully sealed connector was used to make the low-voltage electrical connections. Finally, a Teflon $^{\circledR}$ umbilical cable was used to carry the signals out of the surgical field. All components are gas sterilizable using the conventional Ethylene Oxide gas sterilization protocol.

3. Provision for Monitoring Bending Loads: Because the forces commonly applied by the retractor during laparoscopic surgery $(0-10 \mathrm{~N})$ create bending in the instrument shaft, we used strain gages to evaluate surface strain and serve as force sensors.

4. Depth and Rotation Insensitivity: To accomplish this, it is necessary to measure strain in the two dimensions orthogonal to the long axis of the instrument. Accordingly, one full-bridge is dedicated to each of the orthogonal dimensions so that the net load could be computed as the vector sum of the two component loads.

5. Monotonic Response: Full bridge strain gage configurations satisfy this requirement, and our calibration results affirm compliance.

6. Intra-abdominal Force Measurement: In order to accomplish this, we need to apply the strain gages to the shaft at a point that will lie inside the abdominal wall during most laparoscopic procedures. We opted to isolate the distal shaft to model it as a cantilevered beam. With respect to the strain gages, we made the distal shaft much smaller in cross-sectional area than the proximal shaft. This accomplishes two things: it isolates the distal shaft so that it approximates a cantilevered beam, and it maximizes the telescopic workspace of the device, allowing the device to be extracted further out of the trochar without reducing its intra-abdominal force-sensing capability. 


\subsection{Implementation}

In order to limit the biocompatibility burden to only our device, we used an existing laparoscopic device as the backbone for our project. We adopted a reusable U.S. Surgical $5 \mathrm{~mm}$ laparoscopic retractor as the predicate device and developed a compatible force-sensing sleeve. The U.S. Surgical device is an assembly of 4 separate components: two concentrically loaded stainless steel shafts (S.S. Grade 304), a plastic instrument handle, and a plastic nut for securing the assembly. The primary benefits of this device are its reusability and its narrow shaft that affords us room to develop a force-sensing sleeve without precluding the use of standard trans-abdominal trochars.

Based on the device dimensions presented above, we designed and developed a force-sensing sleeve using Grade 304 Stainless Steel to approximate a homogenous, solid shaft. The sleeve was designed to slip concentrically over the two-component shaft of the U.S. Surgical device. Figure 1 illustrates the device with relevant dimensions. Figure 2 shows an image of the actual device with the distal section of the Teflon® sheath removed.

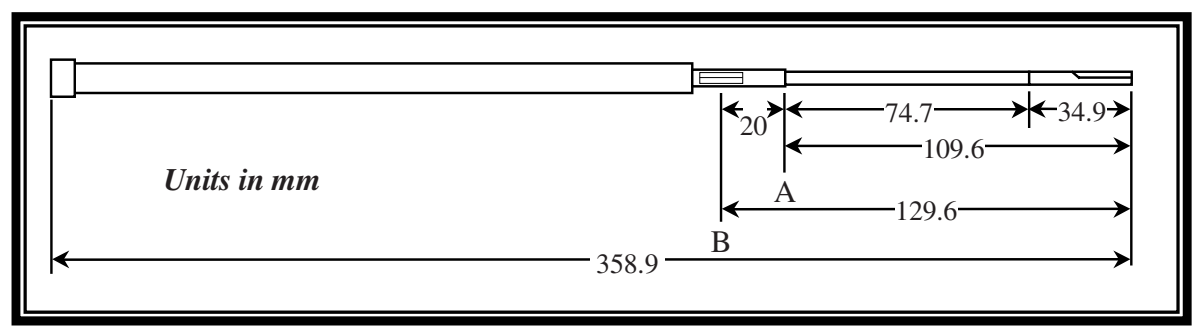

Fig. 1. Assembled Device Dimensions

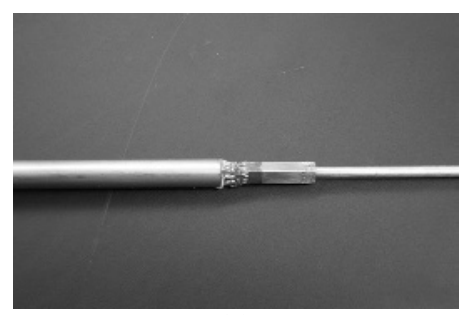

(a)

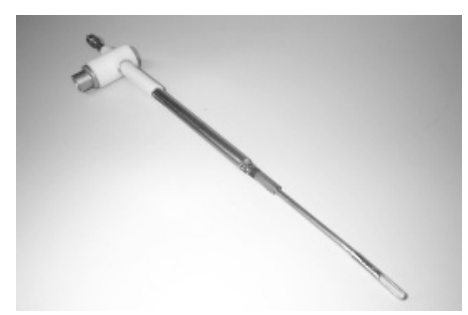

(b)

Fig. 2. (a) Close-up of device strain gages. (b) Full device without sheath.

We incorporated several additional design constraints to permit biocompatibility and sterilization under Ethylene Oxide. The strain gages have been sealed with a biocompatible silicon coating, all leads are Teflon coated conductors that are also sealed with silicon, and the stainless steel shaft is encased in a Teflon sleeve that fully encloses the strain gauges and wiring. The remaining joints are sealed with biocompatible USP Class VI compliant epoxy (Master Bond EP21LV), and the distal free space is filled with a thicker, flexible preparation of the same epoxy. Fully-sealed, leak-tested Fischer ${ }^{\circledast}$ Connectors were used to relay electrical signals across Tefloninsulated composite cables. 


\subsection{Device Calibration}

Our device incorporates independent strain gage bridges for each of the two degrees of freedom, so calibration was performed independently for each. Due to mechanical imperfections, contact between the shafts varied according to load and orientation. Therefore, calibrations were computed independently for each deflection direction for each channel. For each calibration, masses were incrementally applied to the instrument tip, and amplified bridge output voltages were recorded until analog-to-digital conversion saturation occurred. Graphs of the logged data were used to characterize the relationships between bridge output and load. In light of the inherent linearity between load and bridge output, we chose to model the calibration curves as piecewise linear relationships. Figure 3 illustrates the raw data and the division of positive deflection data into individual linear segments. The transitions between the segments can be explained by evaluating the mechanical behavior of the device. Our device effectively has two concentric shafts, with loads applied to the inner shaft and strains recorded on the outer sleeve. The sleeve I.D. is 0.001" larger than the inner shaft O.D., despite attempts at higher fabrication tolerances. Also, there is likely to be irregularity in the sleeve I.D. as a consequence of sub-optimal fabrication technique. Calibration nonlinearity can be explained by irregular contact between the two shafts: for small loads, the inner shaft bends with very limited transmission of load and moment to the outer sleeve - we refer to this condition as "pre-contact"; for large loads, both load and moment are transmitted fully - we refer to this condition as "fullcontact." Surface irregularity creates a third condition, "mid-contact," where load increments change the degree of load and moment transmission. Figure 3 illustrates a three-condition interpolation. In light of the underlying mechanical rationale, we chose to use the three-condition model for all of our calibrations.

The datasets conformed very well to the three-zone model with each linear segment. achieving a mean $\mathrm{R}^{2}$ value of 0.9991 . In order to assess device accuracy using these calibration functions, we applied 10 random loads to each channel and measured our device accuracy. These results are presented for each axis in Table 1.

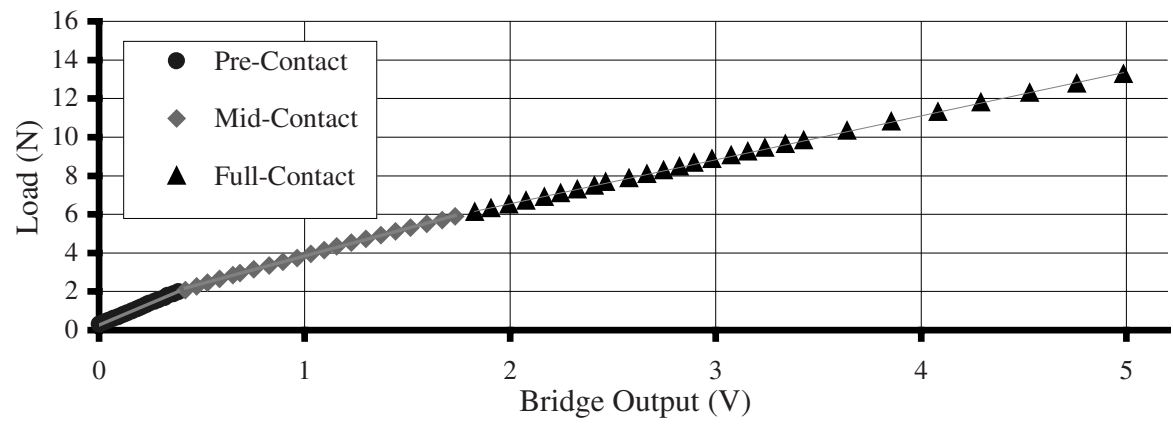

Fig. 3. Sample calibration curve and the three-condition interpolation. 


\section{Application of Device}

To confirm proper operation and collect a sample data set, we recorded the output of the device during retraction of the liver in an in vivo porcine model. Two motions are presented in Figure 4: continuous retraction is presented in Figure 4a and a repetitive retraction motion is shown in Figure $4 \mathrm{~b}$.

Table 1. Device Accuracy

A primary goal of the device, as applied to freehand laparoscopic tools, is to allow sensory substitution to the surgeon to compensate for lost haptic information. We opted to couple our force-sensing device to an audio-feedback apparatus so that we could deliver sensory substitution easily and uniformly. The data acquired through

\begin{tabular}{|c|c|c|c|c|}
\hline \multirow{2}{*}{\multicolumn{2}{|c|}{ Axis }} & \multicolumn{3}{|c|}{ Error } \\
\cline { 3 - 3 }$x$ & \multicolumn{2}{|c|}{ Average } & $\begin{array}{c}\text { St. } \\
\text { Dev }\end{array}$ \\
\hline \multirow{3}{*}{$x$} & Negative & $7.22 \%$ & \multirow{2}{*}{$4.9 \%$} & $6.63 \%$ \\
\cline { 2 - 3 } \cline { 5 - 5 } & Positive & $2.63 \%$ & & $2.90 \%$ \\
\hline \multirow{2}{*}{$y$} & Negative & $2.67 \%$ & \multirow{2}{*}{$3.7 \%$} & $3.41 \%$ \\
\cline { 2 - 3 } \cline { 5 - 5 } & Positive & $4.78 \%$ & & $5.18 \%$ \\
\hline
\end{tabular}
our force-sensing sheath was fed through a SoundBlaster-16 sound card to conventional personal computer speakers. We tested two methods of displaying the magnitude of the force: amplitude modulation and frequency modulation. Preliminary observations were that frequency modulation was easier for users to interpret. However, surgeons expressed concern about continual auditory signals in an operating room already noisy due to verbal communication, music, and sounds from other medical instrumentation.

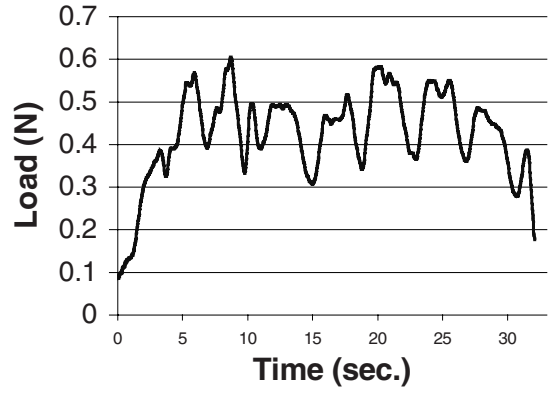

(a)

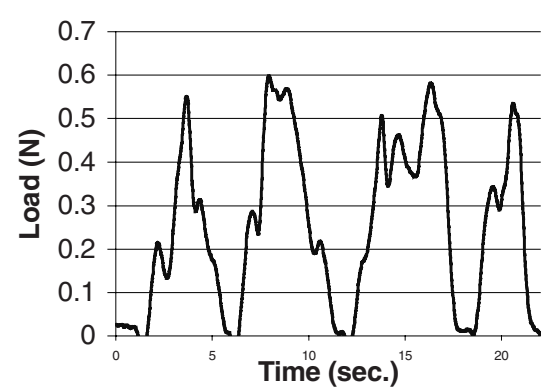

(b)

Fig. 4. (a) Sample Force data for a single retraction. (b) Sample force data for repeated retractions.

\section{Future Directions}

The force-sensing device that we developed is a reasonable first-generation forcesensing instrument. To achieve widespread clinical utility, future generations would have to demonstrate greater sophistication, including the following attributes and enhancements: 
1. Point-Load Insensitivity

2. Axial-Load and Torsion Measurement Provision

3. Complete and Uniform Moment and Load Transfer

4. Incorporation of smaller silicon strain gages

5. Embedded Sensors and leads

In addition, this device can readily be used in the development of surgical feedback and control models. Accordingly, we can quantitatively assess the relative values of different feedback modalities. We can also experiment with simultaneous collection of force and physiological information so that we can correlate force application with tissue response. Another application would be the use of force information as a metric of surgical skill and develop learning curve models using this data.

\section{Conclusions}

Minimally invasive surgery has become the standard of care in many operative procedures performed today. Although MIS allows significantly less patient morbidity than traditional alternatives, it imposes significant hindrances to the surgeon's dexterity and sensory experience. We developed a 2-DOF force-sensing laparoscopic instrument as a first step towards restoring afferent information to the MIS environment. Our device is capable of recording intra-abdominal forces, which can then be displayed to the user through acoustic signals. Moreover, it was developed as a modular device, which affords versatility to even the first generation device. This device can immediately be incorporated into ongoing animal experiments to experimentally validate the device design. We expect that it can be submitted for IRB approval and used to collect human intra-abdominal force information during common general surgical procedures. As minimally invasive surgery takes root, it is clear that it has widespread potential in a variety of surgical disciplines. Force-sensing devices represent a promising contribution to surgical technology by extending the scope of MIS into surgical arenas that critically require delicate afferent information. Heightened intra-operative information promises to enhance the operative experience, improve surgical performance and fuel the advance of Minimally Invasive Surgery.

\section{References}

1. Bholat OS, Haluck RS, Murray WB, Gorman PJ, Krummel TM. Tactile Feedback is Present During Minimally Invasive Surgery. Journal of the American College of Surgeons 1999; 189: 349-355.

2. Brouwer I, Ustin J, Bentley L, Sherman A, Dhruv N, Tendick F. Measuring In Vivo Animal Soft Tissue Properties for Haptic Modeling in Surgical Simulation. Proc. of Med. Meets Virtual Reality 2001; 69-74.

3. Brown JD, Rosen J, Moreyra M, Sinanan M, Hannaford B. Computer-Controlled Motorized Endoscopic Grasper for In Vivo Measurement of Soft Tissue Biomechanical Characteristics.

4. deVisser H, Heijnsdijk AM, Herder JL, Pistecky PV. Forces and Displacements in Colon Surgery. Surgical Endoscopy 2002; 16: 1426-1430.

5. Dubois P, Thommen Q, Jambon AC. In Vivo Measurement of Surgical Gestures. IEEE Transactions on Biomedical Engineering 2002; 49(1): 49-54. 
6. Hyltander A, Liljegren E, Rhodin PH, Lönroth H. The Transfer of Basic Skills Learned in a Laparoscopic Simulator to the Operating Room. Surgical Endoscopy2002; 16: 1324-1328.

7. Poulose BK, Kutka MF, Mendoza-Sagaon M, Barnes AC, Yang C, Taylor RH, Talamini MA. Human vs. Robotic Organ Retraction during Laparoscopic Nissen Fundoplication. Surg Endoscopy 1999; 13: 461-465.

8. Richards C, Rosen J, Hannaford B, Pellegrini C, Sinanan M. Skills Evaluation in Minimally Invasive Surgery using Force/Torque Signatures. Surgical Endoscopy 2000; 14:791798.

9. Rogers DA, Regehr G, Howdieshell TR, Yeh KA, Palm E. The Impact of External Feedback on Computer-Assisted Learning for Surgical Technical Skill Training. American Journal of Surgery 2000; 179: 341-343.

10. Rosen J, Brown JD, Chang L, Barreca M, Sinanan M, Hannaford B. The BlueDRAGON A System for Measuring the Kinematics and the Dynamics of Minimally Invasive Surgical Tools In-Vivo. Proceedings of IEEE ICRA, Washington, DC, May 2002.

11. Rosen, J, Hannaford B, MacFarlane MP, Sinanan MN. Force Controlled and Teleoperated Endoscopic Grasper for Minimally Invasive Surgery - Experimental Performance Evaluation. IEEE Transactions on Biomedical Engineering 1999; 46(10): 1212-1221.

12. Rosen J, MacFarlane M, Richards C, Hannaford B, Sinanan M. Surgeon-Tool Force/Torque Signatures - Evaluation of Surgical Skills in Minimally Invasive Surgery. Proceedings of Medicine Meets Virtual Reality, San Francisco, CA, January 1999.

13. Rosen J, Solazzo M, Hannaford B, Sinanan M. Task Decomposition of Laparoscopic Surgery for Objective Evaluation of Surgical Residents' Learning Curve Using Hidden Markov Model. C.A.S. 2002; 7: 49-61.

14. Smith CD, Farrell TM, McNatt SS, Metreveli RE. Assessing Laparoscopic Manipulative Skills. American Journal of Surgery 2001; 181: 547-550.

15. Wagner, CR, Stylopoulos N, Howe R. The Role of Force Feedback in Surgery: Analysis of Blunt Dissection. Proceedings of Tenth Symposium on Haptic Interfaces for Virtual Environment and Teleoperator Systems, Orlando, FL, March 2002. 